\title{
Bioestimulação e Comportamento Reprodutivo de Novilhas de Corte ${ }^{1}$
}

\author{
Sergio Augusto Ferreira de Quadros², José Fernando Piva Lobato ${ }^{3}$
}

\begin{abstract}
RESUMO - O objetivo do presente trabalho foi determinar se a bioestimulação é capaz de alterar favoravelmente o comportamento reprodutivo de novilhas de corte em sua primeira estação reprodutiva. Para isso, 50 dias antes do início da inseminação artificial (IA), 60 novilhas de dois anos (Hereford x Nelore) foram divididas ao acaso em dois grupos: bioestimuladas (BE), através do uso de rufiões, ou não (NE). As taxas de novilhas cíclicas antes do início da IA foram de 76 e 56\% para BE e NE, respectivamente. As taxas de prenhez foram de 90 e $73 \%$ para BE e NE, respectivamente. A bioestimulação determinou diferenças nos percentuais acumulados de novilhas inseminadas por subperíodos de 21 dias de IA, sem alterar a data média de concepção. Foi observado efeito de idade/desenvolvimento corporal na resposta à bioestimulação.
\end{abstract}

Palavras-chave: bioestimulação, comportamento reprodutivo, efeito do macho, novilhas

\section{Biostimulation and Reproductive Performance of Beef Heifers}

\begin{abstract}
The goal of this experiment was to evaluate if biostimulation may favourably change the reproductive performance of beef heifers on their first breeding season. For this purpose, 50 days before the artificial insemination (AI) period, 60 two-year-old heifers (Hereford $\mathrm{x}$ Nelore) were randomly distributed into two treatment groups: biostimulatated (BE) using a ruffian or not biostimulated (NE). The rates of cycling heifers before AI were 76 and 56\% for BE e NE, respectively. Conception rates were 90 and $73 \%$ for BE e NE, respectively. Biostimulation changed accumulated percents of inseminated heifers by the end of 21 days periods during AI, without changing mean conception day. It was observed the effect of age/body development in the responses to biostimulation.
\end{abstract}

Key Words: biostimulation, heifers, male-effect, reproductive performance

\section{Introdução}

Altas taxas reprodutivas são fundamentais na obtenção de elevada eficiência nos sistemas de produção de bovinos de corte. No Brasil, as baixas taxas de natalidade são conseqüência, fundamentalmente, do prolongado anestro pós-parto, fenômeno de especial relevância nas vacas de primeira cria. Trabalhando com estação reprodutiva fixa, é lógico constatar que quanto mais cedo a vaca vier a parir, mais tempo vai dispor para voltar a ciclar até o início do próximo período de acasalamento. Assim, em um mesmo nível nutricional, as vacas cujos partos ocorrem mais cedo têm maior chance de reconcepção (Reynolds, 1973). Lesmeister et al. (1973) determinaram que novilhas que concebem cedo no seu primeiro acasalamento e, portanto, vêm a parir nos primeiros dias da estação de nascimento do ano seguinte, desmamam bezerros mais pesados e em maior número ao longo de sua vida produtiva.
Para cumprir este propósito, as novilhas devem ter uma concepção precoce durante sua primeira estação reprodutiva. Para atingir esta meta, a maioria das novilhas deve estar ciclando regularmente já ao início desta estação. Como a nutrição tem efeito decisivo sobre este evento, a obtenção do peso crítico de acasalamento para as novilhas é medida prioritária para o sucesso reprodutivo desta categoria (Lobato, 1997 ). Entretanto, outro fator ambiental que pode ter efeito positivo sobre o percentual de novilhas que inicia a estação reprodutiva ciclando é a bioestimulação ou o "efeito - touro".

As fêmeas dos mamíferos, especialmente dos que vivem em grandes grupos, são envoltas em um ambiente social muito rico e complexo, cheio de estímulos sensitivos provenientes dos demais componentes do grupo e, muito especialmente, de suas crias e potenciais parceiros sexuais. Quando percebidos, estes estímulos desencadeiam mudanças em muitos processos fisiológicos e comportamentais, incluindo

\footnotetext{
1 Parte da tese de Doutorado do primeiro autor apresentada à UFRGS.

2 Professor do Departamento de Zootecnia e Desenvolvimento Rural, CCA - UFSC. E.mail: safq@uol.com.br

3 Professor do Departamento de Zootecnia, Fac. Agronomia - UFRGS. E.mail: jose.fernando.lobato@ufrgs.br
} 
aqueles relacionados à reprodução (Martin, 2002).

A separação de machos e fêmeas, formando grupos distintos nos sistemas criatórios como regra de manejo, pode ter suprimido o "efeito do macho", presente em comunidades ferais. Assim, o reagrupamento dos sexos em períodos estratégicos pode ser vantajoso no sentido de maximizar a performance reprodutiva.

Berardinelli et al. (1978) e MacMillan et al. (1979) não encontraram diferença na idade à puberdade de novilhas expostas ou não ao touro, enquanto Izard \& Vandenbergh (1982) evidenciaram efeito positivo no uso de urina de touro em tratamentos oronasais na antecipação da puberdade de novilhas. Em experimento fatorial com as variáveis bioestimulação (BE) ou não (NE) e ganho diário médio de 0,8 kg/dia (HG) ou 0,6 kg/ dia (MG), Roberson et al. (1991) observaram que as novilhas do tratamento BE-HG atingiram a puberdade com menor idade e menor peso. As novilhas dos tratamentos BE-MG e NE-HG tiveram idades à puberdade equivalentes, o que significou que as novilhas BE-MG atingiram-na com menor peso. As mais tardias foram as novilhas NE-MG. Em Santa Vitória do Palmar, Rio Grande do Sul, trabalhando com novilhas Aberdeen Angus de dois anos de idade, Assis et al. (1998) concluíram que a bioestimulação nos 75 dias que antecediam o acasalamento antecipou a maturidade sexual. Entretanto, Rosa (1999), na mesma região, não observou efeito sobre maturidade sexual, freqüência de estro e índice de prenhez de novilhas Angus e Hereford de 18 meses de idade, mas melhor índice de concepção ao primeiro serviço.

O presente trabalho foi conduzido com o objetivo de determinar se a presença do macho por um período de 50 dias antes do início da primeira temporada reprodutiva (inseminação artificial) de novilhas de corte é capaz de alterar favoravelmente seu comportamento reprodutivo.

\section{Material e Métodos}

O experimento foi conduzido na Fazenda Capivarita, município de Pantano Grande, Rio Grande do Sul. Foram utilizadas 60 novilhas cruzas Hereford x Nelore do rebanho da propriedade com média de 24,5 meses de idade, distribuídas nos seguintes tratamentos:

BE - 30 novilhas em potreiro de aveia e azevém junto a rufiões (machos bioestimuladores) por 50 dias.
NE - 30 novilhas em potreiro com condição forrageira semelhante, sem a presença de macho, durante o mesmo período.

Como rufiões, foram utilizados touros da raça Jersey com desvio de pênis, na proporção de 26 fêmeas para 1 macho (considerando as vacas com cria pertencentes a outra fase deste projeto que estavam locadas no mesmo potreiro). Durante o período de inseminação artificial (10/10/2000 a 11/12/2000), todas as novilhas e rufiões passaram a outro potreiro, sendo manejadas em conjunto. Todas as novilhas foram inseminadas pelo mesmo técnico, que usou os mesmos procedimentos.

Durante o período experimental, os animais foram observados duas vezes ao dia para a detecção de cio (das 6 h30 às 7 h30 e das 18h30 às 19h30), procedimento que foi repetido durante o período de inseminação artificial. No entanto, para análise estatística, foram considerados somente os dados relativos às determinações de progesterona. Para tal, foram feitas coletas semanais de sangue da jugular um mês antes de iniciar a inseminação artificial (dias 12, 19, 26 de setembro e 04 de outubro). Em torno de 6 a 8 horas após, quando as amostras apresentavam coagulação, o soro foi retirado e congelado. A determinação de progesterona (P4) foi feita pela técnica de radioimunoensaio (RIA) em laboratório do ICTA-UFRGS, utilizando kit da DPC medlab. Foi considerada cíclica toda novilha cuja dosagem sérica de progesterona ultrapassou $1 \mathrm{ng} / \mathrm{mL}$ em determinada amostra. As novilhas foram pesadas e avaliadas quanto à condição corporal, usando escores de 1 a 5 (Lowman et al., 1973), no início do período experimental (16/08), início das tomadas de sangue (12/09), final da bioestimulação (04/10) e final da inseminação (06/12).

O delineamento utilizado foi o inteiramente casualizado. Os dados foram submetidos à análise da variância utilizando o procedimento GLM do SAS (SAS, 1996). Os modelos incluíram os efeitos de tratamento, variação no escore de condição corporal, peso ao início da estação reprodutiva, idade e interação tratamento $\mathrm{x}$ idade, que, conforme sua significância para a variável em questão, foram ou não destacados do erro experimental. O número de novilhas cíclicas em cada semana que antecedeu o período de reprodução e a quantidade inseminada em cada subperíodo de 21 dias de temporada reprodutiva foram submetidos ao teste Exato de Fisher, segundo Gomes (1990). 


\section{Resultados e Discussão}

A Tabela 1 apresenta o desenvolvimento ponderal das novilhas durante o período experimental, utilizando-se as médias de peso e escore de condição corporal calculadas pelo método dos quadrados mínimos. No decorrer deste período, as novilhas BE sofreram certa restrição no ganho de peso porque a aveia do potreiro em que foram locadas completou seu ciclo e o desenvolvimento do azevém foi muito escasso. Conseqüentemente, houve diferença $(p=0,0002)$ entre os ganhos diários médios no período de $16 / 8$ a 04 / 10 (período de bioestimulação) entre os tratamentos, observando-se 0,730 e 0,972 kg/dia para BE e NE, respectivamente. Ao iniciar o período de inseminação artificial, os pesos médios das novilhas foram de 315,06 e $327,80 \mathrm{~kg}$ para os tratamentos BE e NE, respectivamente $(\mathrm{p}=0,06)$.

Apesar desta involuntária pior condição nutricional proporcionada às novilhas $\mathrm{BE}$, ao iniciar o período reprodutivo (IA) $76 \%$ destas novilhas já estavam ciclando, enquanto somente $56 \%$ das novilhas NE o fizeram $(p=0,06)$. Como a análise da variância evidenciou efeito de idade $(\mathrm{p}=0,12)$ sobre esta variável, procedeu-se, para fins de análise estatística, à subdivisão dos tratamentos em dois lotes de igual número de unidades amostrais $(\mathrm{n}=15)$ por data de nascimento. Os subgrupos apresentavam, no início do experimento, as idades médias de 25,5 meses para as novilhas mais velhas e 23,5 meses para as mais jovens, com pesos médios de 289,3 e 268,4 kg $(\mathrm{p}=0,005)$, respectivamente. Pode-se perceber que a diferença na taxa de novilhas ciclando entre os tratamentos foi maior nas novilhas mais velhas: $\mathrm{BE}=93 \%$ $x \mathrm{NE}=60 \%(\mathrm{p}=0,03)$ e não significativo nas mais jovens: $\mathrm{BE}=60 \% \times \mathrm{NE}=53 \%(\mathrm{p}=0,65)$.

As taxas de prenhez resultantes dos 63 dias de inseminação artificial foram de 90 e $73 \%$ para os grupos $\mathrm{BE}$ e $\mathrm{NE}$, respectivamente $(\mathrm{p}=0,07)$. A mesma subdivisão feita por idade foi testada também para esta variável. Entre as novilhas mais velhas, as taxas de prenhez foram de 86 e $80 \%(p=0,84)$ e para as mais jovens de 93 e $66 \%(\mathrm{p}=0,05)$ para $\mathrm{BE}$ e NE, respectivamente.

Estes resultados podem ser explicados de acordo com o tempo em que foram obtidos. Ao analisar a variável taxa de novilhas ciclando antes da inseminação artificial, a maior resposta à bioestimulação ocorreu entre o grupo de novilhas mais velhas, portanto mais próximas da puberdade. As novilhas mais jovens, de menor desenvolvimento corporal, possivelmente ainda apresentavam "bloqueio" à completa atividade do eixo hipotálamo-hipófise-gonadal, que não pôde ser superado pela bioestimulação neste período. Com o passar do tempo e a aproximação da maturidade fisiológica, este "bloqueio" foi superado já durante o período de inseminação. Iniciado este período. Entre o grupo mais jovem, as novilhas BE passaram a apresentar cios mais precocemente e, conseqüentemente, terminaram os 63 dias de inseminação artificial com maior taxa de prenhez ( $\mathrm{p}=0,05)$. Já as boas taxas de prenhez entre ambos os tratamentos nas novilhas mais velhas indicam que, independentemente do tratamento no período anterior (bioestimulação ou

Tabela 1 - Pesos e escores de condição corporal (ECC) ao longo do período experimental

Table 1 - Liveweights and body condition scores (BCC) during experimental period

\begin{tabular}{|c|c|c|c|c|}
\hline \multirow[t]{2}{*}{$\begin{array}{l}\text { Data/evento } \\
\text { Date/event }\end{array}$} & \multicolumn{2}{|c|}{$\begin{array}{c}\text { Peso (kg) } \\
\text { Liveweight }(\mathrm{kg})\end{array}$} & \multicolumn{2}{|c|}{$\begin{array}{l}\text { ECC } \\
B C C\end{array}$} \\
\hline & $\mathrm{BE}$ & $\mathrm{NE}$ & $\mathrm{BE}$ & $\mathrm{NE}$ \\
\hline $\begin{array}{l}\text { 16/8 - Início dos tratamentos } \\
\text { Beginning of treatments }\end{array}$ & 278,53 & 279,00 & 3,03 & 3,03 \\
\hline $\begin{array}{l}12 / 9-1^{\mathrm{a}} \text { amostra de sangue } \\
\text { First blood sample }\end{array}$ & 302,80 & $317,26 a$ & 3,15 & 3,23 \\
\hline $\begin{array}{l}\text { 4/10 - Início da IA } \\
\text { Beginning of } A I\end{array}$ & 315,06 & $327,80 \mathrm{~b}$ & 3,20 & 3,22 \\
\hline $\begin{array}{l}\text { 6/12 - Fim da IA } \\
\text { End of AI }\end{array}$ & 336,20 & $348,27 c$ & 3,08 & 3,11 \\
\hline
\end{tabular}

Médias de peso seguidas de letras na mesma linha apresentam: a) $p=0,02, b) p=0,06$ e c) $\mathrm{p}=0,10$.

Means followed byt letters in the same row show: a) $p=.02, b) p=.06$ and c) $p=.10$

R. Bras. Zootec., v.33, n.3, p.679-683, 2004 
não), apresentavam, durante a estação reprodutiva, condições de desenvolvimento fisiológico que lhes permitiram atingir estes índices.

É razoável considerar que parte destas novilhas já havia atingido a puberdade no outono anterior e entrado em anestro durante o inverno, devido às limitações nutricionais neste período. Entretanto, mesmo que ambos os fatores (pré-puberdade ou anestro nutricional) tenham determinado ausência de atividade lútea, é de se esperar que a influência do fator insuficiência de maturidade fisiológica para atingir a puberdade tenha sido maior no grupo de novilhas mais jovens.

Os resultados obtidos por Izard \& Vanderbergh (1982) dão suporte a essas inferências. Estes autores, ao objetivarem a indução da puberdade em novilhas pela utilização da urina de touro, observaram que a possibilidade de resposta ao estímulo foi dependente do desenvolvimento corporal (peso) das novilhas (assim como, no presente trabalho, as novilhas mais velhas apresentaram maior resposta em termos de taxa de cios).

A Figura 1 apresenta a taxa acumulada de novilhas cíclicas por tratamento no mês que antecedeu a inseminação artificial. Percebe-se que, na primeira semana de avaliação (12/9), ambos os tratamentos tinham taxas de cio muito próximas (33\% x 30\%, $\mathrm{p}=0,21$ ), com as diferenças se acentuando a partir da segunda ( $60 \%$ x $36 \%, \mathrm{p}=0,04)$, mantendo-se elevadas na terceira $(73 \%$ x $46 \%, \mathrm{p}=0,02)$ e quarta $(76 \% \mathrm{x}$ $56 \%, \mathrm{p}=0,06)$ semanas.

O percentual acumulado de novilhas inseminadas por subperíodo de 21 dias é apresentado na Figura 2.

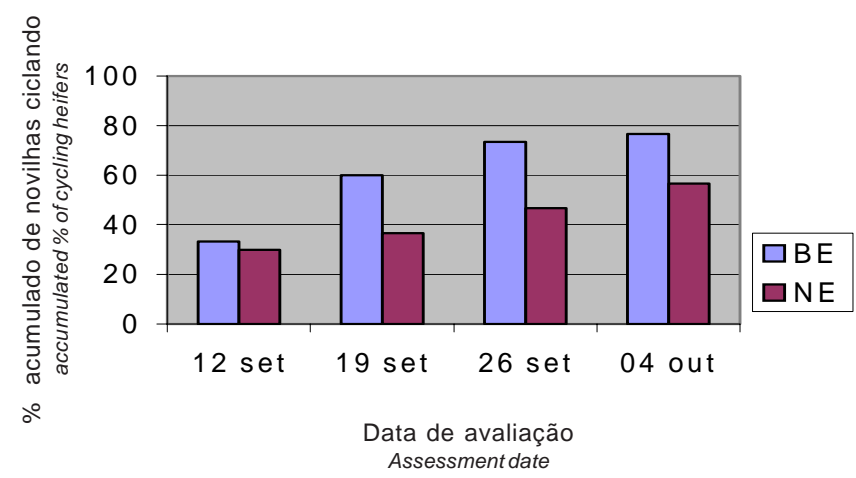

Figura 1 - Efeito da bioestimulação sobre a atividade estral de novilhas de corte.

Figure 1 - Effect of biostimulation on estrual activity of beef heifers.

R. Bras. Zootec., v.33, n.3, p.679-683, 2004
Pode-se observar que já nos primeiros 21 dias o total de novilhas inseminadas foi elevado em ambos os tratamentos, com taxas de 70 e $63 \%$ para as novilhas $\mathrm{BE}$ e NE, respectivamente $(\mathrm{p}=0,18)$. A diferença se acentuou no segundo subperíodo de 21 dias com as taxas de $83 \%$ para $\mathrm{BE}$ e $66 \%$ para $\mathrm{NE}(\mathrm{p}=0,08)$, culminando com $90 \%$ (BE) e $73 \%$ (NE) apresentados ao final dos 63 dias de temporada reprodutiva $(\mathrm{p}=0,07)$.

Apesar das diferenças entre os tratamentos encontradas na taxa de natalidade e nos subperíodos da temporada de inseminação, ambos tiveram como data média de inseminação o dia 26 de outubro, ou seja, as novilhas de ambos os tratamentos foram inseminadas, em média, 16 dias após o início da temporada reprodutiva. Esta observação pode ser explicada por dois fatores. Primeiro, porque nesta análise as novilhas que não ciclaram (e, portanto, não foram inseminadas) não foram incluídas; segundo, devido ao reduzido período de inseminação utilizado. Se este período tivesse se prolongado por 90 dias ou mais, dar-se-ia chance para que as novilhas que até então não haviam ciclado o fizessem. Com isso, reduz-se-ia a diferença entre as taxas de natalidade, mas seriam permitidas concepções mais tardias e, assim, poderia surgir diferença na data média de inseminação artificial entre os tratamentos. Estes resultados podem ser comparados com os obtidos por Izard \& Vanderbergh (1982). Estes autores, ao final dos 90 dias de monta natural que sucederam os tratamentos, não encontraram diferença significativa entre as taxas de prenhez e atribuiram esta condição ao prolongado período reprodutivo utilizado. De for-

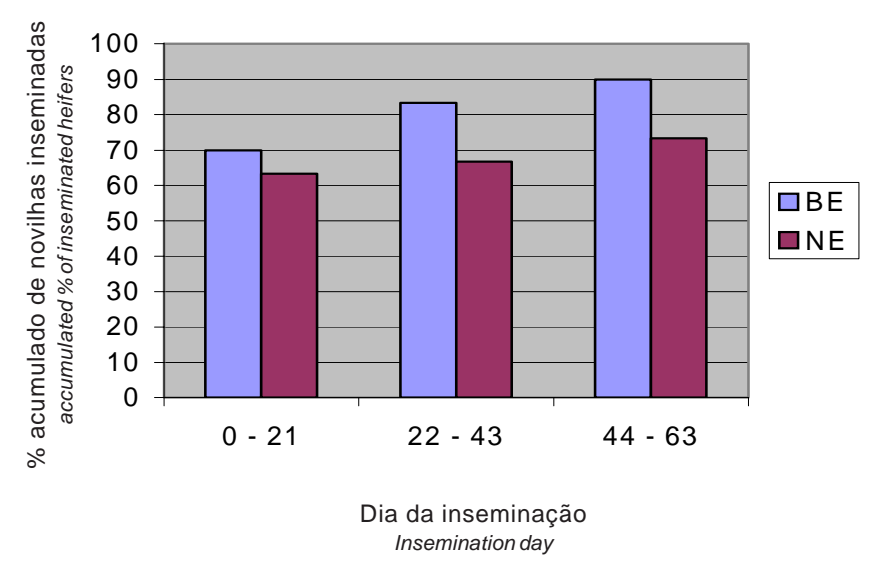

Figura 2 - Efeito da bioestimulação sobre o subperíodo de inseminação artificial.

Figure 2 - Effect of biostimulation on artificial insemination subperiod. 
ma semelhante, Roberson et al. (1991) não encontraram diferença na taxa de prenhez ao longo de toda temporada reprodutiva, mas as novilhas bioestimuladas apresentaram melhores índices nos 21 dias de inseminação artificial que antecederam os 30 dias de monta natural.

Com relação à idade, as novilhas mais velhas foram inseminadas, em média, 7 dias mais cedo que as mais jovens $(\mathrm{p}=0,06)$, possivelmente como conseqüência da já referida maior taxa de fêmeas ciclando ao iniciar a temporada reprodutiva neste subgrupo. Reforça essa assertiva o fato de se ter evidenciado, para as novilhas mais jovens, maior efeito do peso ao início da inseminação artificial $(\mathrm{p}=0,01)$ para a variável data média de inseminação, se comparado com a importância deste peso para as mais velhas $(p=0,93)$. Pode-se inferir que, para estas, a variável peso teve importância desprezível, porque todas já haviam atingido desenvolvimento corporal adequado para ciclar.

\section{Conclusões}

A bioestimulação por um período de 50 dias determinou maior taxa de novilhas cíclicas antes de se iniciar a temporada reprodutiva $(p=0,06)$, bem como maior taxa de prenhez $(\mathrm{p}=0,07)$.

A presença do macho determinou maiores percentuais acumulados de novilhas inseminadas por subperíodos de 21 dias de IA, sem alterar a data média de concepção.

A resposta das novilhas ao estímulo da presença do machoé dependente da idade/desenvolvimento corporal.

\section{Agradecimento}

Pela receptividade e pela cedência das instalações e dos animais, nossos agradecimentos à direção da Motrisa, na pessoa do Eng. Agrônomo Luiz Sérgio Ruwer. Ao Sr. Gilceu Dalla Giacomazza, administrador da Fazenda Capivarita, pelo apoio durante a execução do experimento. Aos prestimosos companheiros que auxiliaram nos trabalhos, Med.Vet. Paulo Henrique Ferreira de Quadros, Sr. José Francisco Freitas Rodrigues, Sr. André Siwinski e Sr. Ranzoli Moraes, nossa gratidão.

\section{Literatura Citada}

ASSIS, R.R.; PIMENTEL, M.A.; JARDIM, P.O. et al. Efeito da bioestimulação por meio de machos vasectomisados sobre a maturidade sexual de novilhas da raça Aberdeen Angus. In: REUNIÃO ANUAL DA SOCIEDADE BRASILEIRA DE ZOOTECNIA, 36., 1998, Botucatu. Anais... Botucatu: Sociedade Brasileira de Zootecnia, 1998. p.157-159.

BERARDINELLI, J.G.; FOGWELL, R.L.; INSKEEP, E.K. Effect of electrical stimulation or presence of a bull on puberty in beef heifers. Theriogenology, v.9, p.133-139, 1978.

GOMES, F.P. Curso de estatística experimental. 13.ed. São Paulo: Nobel, 1990. 467p.

IZARD, M.K.; VANDERBERGH, J.G. The effects of bull urine on puberty and calving date in crossbred beef heifers. Journal of Animal Science, v.55, p.1160-1168, 1982.

LESMEISTER, J.L.; BURFENING, J.P.; BLACKWELL, R.L. Date of first calving in beef cows and subsequent calf production. Journal of Animal Science, v.31, n.1, p.1-14, 1973.

LOBATO, J.F.P. Sistemas intensivos de produção de carne bovina: I. Cria. In: SIMPÓSIO SOBRE PECUÁRIA DE CORTE, 4., 1996, Piracicaba. Anais... Piracicaba: Fundação de Estudos Agrários Luiz de Queiroz, 1997. p.161-204.

LOWMAN, B.G.; SCOTT, N.; SOMERVILLE, S. Condition scoring beef cattle. Edinburgh: East of Scotland College of Agriculture, 1973. (Bulletin, 6). 8p.

MacMILLAN, K.L.; ALLISON, A.J.; STRUTHERS, G.A. Some effects of running bulls with suckling cows or heifers during the premating period. New Zealand Journal of Experimental Agriculture, v.7, p.1121-1124, 1979.

MARTIN, G.B. Social-sexual signs and reproduction in mammals - an overview. In: CURSO INTERNACIONAL SOBRE FEROMONAS Y BIOESTIMULACIÓN SEXUAL, 1., 2002. Anais... Universidad Nacional Autonoma de Mexico, 2002. p.11-28.

REYNOLDS, W.R. Breeds and reproduction. In:, CUNHA, T.J.; WARNICK, A.C.; KOGER, M. (Eds.) Factors affecting calf crop. 3.ed. Gainsville: Florida Press, 1973. p.244-259.

ROBERSON, M.S.; WOLFE, M.W.; STUMPF, T.T. et al. Influence of growth rate and exposure to bulls on age at puberty in beef heifers. Journal of Animal Science, n.69, p. 2092-2098, 1991.

ROSA, T.S. Influência da bioestimulação através de fêmeas androgenizadas sobre a eficiência reprodutiva de novilhas de corte. Pelotas: Universidade Federal de Pelotas, 1999. 75p. Dissertação (Mestrado em Zootecnia) - Universidade Federal de Pelotas, 1999.

STATISTICAL ANALYSES SYSTEM - SAS. SAS/STAT. User's guide. 11.ed. Cary: 1996. 